\title{
Presença de macrófitas em reservatórios de abastecimento e implicações no tratamento de água
}

\author{
Macrophytes presence in supply reservoirs and its \\ implication in water treatment
}

Data de entrada: $10 / 06 / 2016$

Data de aprovação: 09/05/2017

\section{Resumo}

Este estudo correlaciona a presença de macrófitas aquáticas com variáveis abióticas em reservatórios da Região Metropolitana do Recife-PE. As coletas foram semestrais, realizadas em 2014, em cinco captações. As variáveis abióticas medidas foram: alcalinidade, $\mathrm{pH}$, cálcio, magnésio, condutividade, turbidez, cor, dureza, cloretos, sulfato, amônia, nitrato, nitrito e fósforo. A correlação foi feita por meio do modelo de regressão linear generalizado (MLG) e o coeficiente de Spearman (rho). As espécies encontradas em maior frequência foram a Salvinia auriculata e Pistia stratiotes correlacionadas positivamente com a variável abiótica fósforo. A proliferação das macrófitas nos mananciais tem comprometido as unidades das estações de tratamento de água, sendo necessária a adoção de medidas de proteção das margens dos reservatórios, reduzindo o aporte de nutrientes. Palavras-chave: Plantas aquáticas. Fatores abióticos. Barragem. Poluição.

\section{Abstract}

This study correlates the presence of aquatic macrophytes and abiotic variables in reservoirs of the Recife Metropolitan Region. Samplings were semiannual, during 2014, in five reservoirs. The abiotic variables measured were: alkalinity, pH, calcium, magnesium, conductivity, turbidity, color, hardness, chloride, sulfate, ammonia, nitrate, nitrite and phosphorus. The correlation was analyzed using generalized linear regression models (GLM) and the Spearmancoefficient (rho). Thespeciesfoundmost often weretheSalviniaauriculataand Pistiastratiotespositively correlated with the abiotic variable phosphorus. The proliferation of macrophytes in water sources have compromised units of water treatment plants, it is necessary to adopt protective measures to reduce the supply of nutrients. Keywords: Aquatic Plants. Abiotic factors. Dam. Pollution.

\footnotetext{
Valderice Pereira Alves Baydum - Doutora em Engenharia Química Universidade Federal Pernambuco. Mestre em Engenharia Química Universidade Federal de Pernambuco. Engenheira Ambiental Universidade Católica de Pernambuco e Química Industrial Universidade Federal Pernambuco. Professora da Universidade Católica de Pernambuco. Analista em Saneamento Química (COMPESA). com.br.

Fábio Henrique Portella Corrêa de Oliveira - Doutor em Botânica Universidade Federa Rural Pernambuco, Mestre em Biotecnologia de Produtos Bioativos Universidade Federal de Pernambuco, Bacharel em Ciências Biológicas Universidade de Pernambuco. Professor da Faculdade São Miguel. Analista Gestão Biólogo (COMPESA). 


\section{INTRODUÇÃO}

As macrófitas são plantas que apresentam grande capacidade de adaptação e amplitude ecológica, habitando ambientes de águas doce, salobra e salgadas, de água estacionária e corrente. Possuem funções ecológicas importantes, mas em elevadas densidades podem provocar impactos na qualidade da água, no processo de captação e no assoreamento da represa (SILVA, 2011). Com o assoreamento aumenta a acumulação de vegetação, o lago se torna cada vez mais raso, podendo vir a desaparecer (VON SPERLING, 2005). Esse efeito tem sido observado na Barragem Tapacurá (Figura 1), manancial que abastece a região metropolitana do Recife-PE, onde há predominantemente ilhas de Pistia stratiotes.

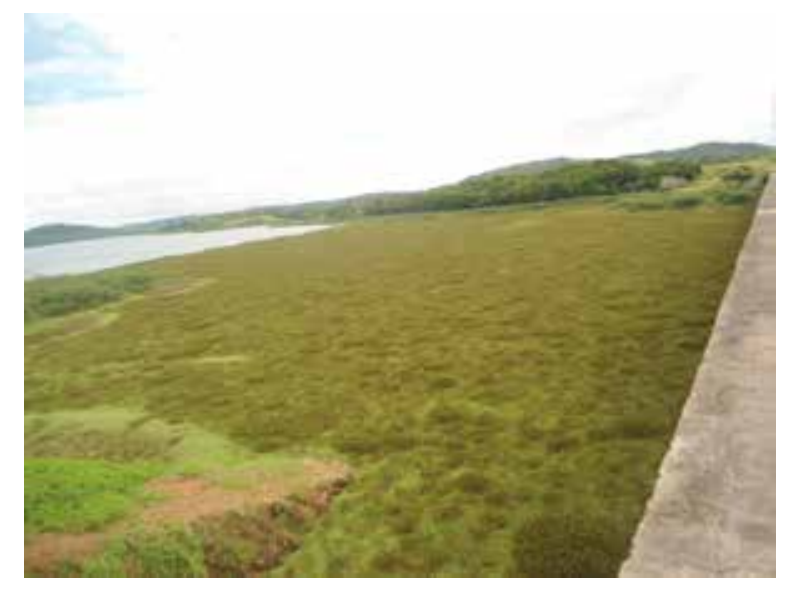

Figura 1: Presença de plantas marginais e flutuantes na Barragem de Tapacurá - São Lourenço da Mata/PE.

A colonização de um ambiente hídrico por plantas aquáticas é uma indicação de que a água pode apresentar alterações decorrentes da intensificação de atividades antrópicas e normalmente encontra-se associada ao aporte de nutrientes (nitrogênio e fósforo) para o rio, por meio de despejos domésticos, industriais e fertilizantes químicos empregados nos cultivos (BORNETTE e PUIJALON, 2011).

O florescimento de macrófitas decorrentes do aumento da concentração de nutrientes no manancial pode ter efeitos diretos na qualidade da água, como problemas estéticos, recreacionais e até de redução na capacidade de transporte e navegação (VON SPERLING, 2005). Entretanto, alguns trabalhos têm mostrado que espécies de macrófitas possuem capacidade de remover nutrientes da coluna d'água, tais como nitrogênio e fósforo (ALBERTONI et al., 2014; HOLMROOS et al., 2015), bem como metais (MEl e ZHANG, 2013, YADAV et al., 2015), diminuindo a eutrofização de origem antrópica.

Além dos impactos negativos gerados no meio aquático pela eutrofização, Richter e Netto (2005) afirmam que esse impacto se reflete no aumento dos custos para tratamento da água, obstrução dos filtros e aumento do consumo de água para lavagem. Thomaz e Bini (2003) relatam efeitos diretos e indiretos de macrófitas no abastecimento, podendo desencadear alterações na qualidade da água, na operação das estações de tratamento de água (ETA), dos sistemas de reservação e distribuição, aumento do consumo de cloro devido à presença de matéria orgânica e amônia e diminuição da eficiência da desinfecção (DAMATO, 2016, DI BERNARDO, 1995).

O objetivo deste estudo é identificar a influência de parâmetros físico-químicos sobre comunidades de macrófitas em captações de água bruta de reservatórios de abastecimento da região metropolitana do Recife-PE e observar a influência desses organismos no tratamento de água. $O$ conhecimento das macrófitas existentes e as condições abióticas do manancial podem subsidiar propostas de manejo e prevenção de sua proliferação descontrolada e consequências no tratamento de água.

\section{METODOLOGIA}

\section{1 Área de estudo}

As captações de água bruta em estudo estão localizadas na Bacia do Rio Capibaribe e no grupo de bacias de pequenos rios litorâneos 2 - GL 2 (Tabela 1). 
Tabela 1: Descrição dos reservatórios hídricos estudados com respectivos pontos de captação e coordenadas geográficas:

\begin{tabular}{|c|c|c|c|c|}
\hline Bacia & Ponto & Captações & Latitude & Longitude \\
\hline \multirow{4}{*}{ 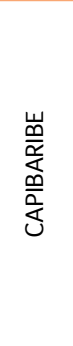 } & 1 & $\begin{array}{l}\text { Castelo - Rio } \\
\text { Capibaribe }\end{array}$ & $7^{\circ} 59^{\prime} 43,95^{\prime \prime S}$ & $35^{\circ} 03^{\prime} 11,88^{\prime \prime} \mathrm{W}$ \\
\hline & 2 & $\begin{array}{l}\text { Tiuma - Rio } \\
\text { Capibaribe }\end{array}$ & $7^{\circ} 58^{\prime} 53,60 " \mathrm{~S}$ & $35^{\circ} 04^{\prime} 39,93^{\prime \prime} \mathrm{W}$ \\
\hline & 3 & $\begin{array}{l}\text { Barragem } \\
\text { Goitá - Rio } \\
\text { Capibaribe }\end{array}$ & $7^{\circ} 58^{\prime} 10,54^{\prime \prime S}$ & $35^{\circ} 06^{\prime} 56,31^{\prime \prime} \mathrm{W}$ \\
\hline & 4 & $\begin{array}{c}\text { Barragem } \\
\text { Tapacurá } \\
\text { - Rio } \\
\text { Capibaribe }\end{array}$ & $8^{\circ} 02^{\prime} 35^{\prime \prime} \mathrm{S}$ & $35^{\circ} 09^{\prime} 45^{\prime \prime} \mathrm{W}$ \\
\hline$\frac{N}{0}$ & 5 & $\begin{array}{c}\text { Barragem } \\
\text { Duas Unas } \\
\text { - Rio Jaboatão }\end{array}$ & $8^{\circ} 05^{\prime} 31,24^{\prime \prime} \mathrm{S}$ & $35^{\circ} 02^{\prime} 18,90^{\prime \prime} \mathrm{W}$ \\
\hline
\end{tabular}

\subsection{Amostragem e análise dos dados}

As coletas foram realizadas mensalmente, entre janeiro e dezembro de 2014. As macrófitas foram coletadas com auxílio de uma rede, enquanto as amostragens de água foram realizadas diretamente em frascos de vidro âmbar na subsuperfície dos reservatórios. As variáveis físico-químicas analisadas foram: alcalinidade, cálcio, magnésio, $\mathrm{pH}$, condutividade, turbidez, cor, dureza, cloretos, sulfatos, amônia, nitrito, nitrato e fosfato de acordo com APHA (2012). A identificação das macrófitas foi realizada de acordo com Lima et al. (2011). Os ensaios foram conduzidos no laboratório de análises físico-químicas e de hidrobiologia da Gerência de Qualidade da Companhia de Saneamento do Estado.

As implicações da presença de macrófitas nas etapas do tratamento de água foram avaliadas in loco em uma ETA localizada na região metropolitana do Recife.

Foram realizadas análises estatísticas não-paramétricas, utilizando os modelos lineares gerais (GLM) para avaliar os parâmetros físico-químicos preditores da presença ou ausência dos táxons de macrófitas nos ambientes estudados. Consideram-se significati- vas as análises com valor de $p<0,05$. Os testes foram executados utilizando o programa Statistica 7.

\section{RESULTADOS E DISCUSSÃO}

Os táxons de macrófitas presentes nas unidades amostrais foram Salvinia auriculata, Eichhornia crassipes, Egeria densa e Pistia stratioides (Figura 2).

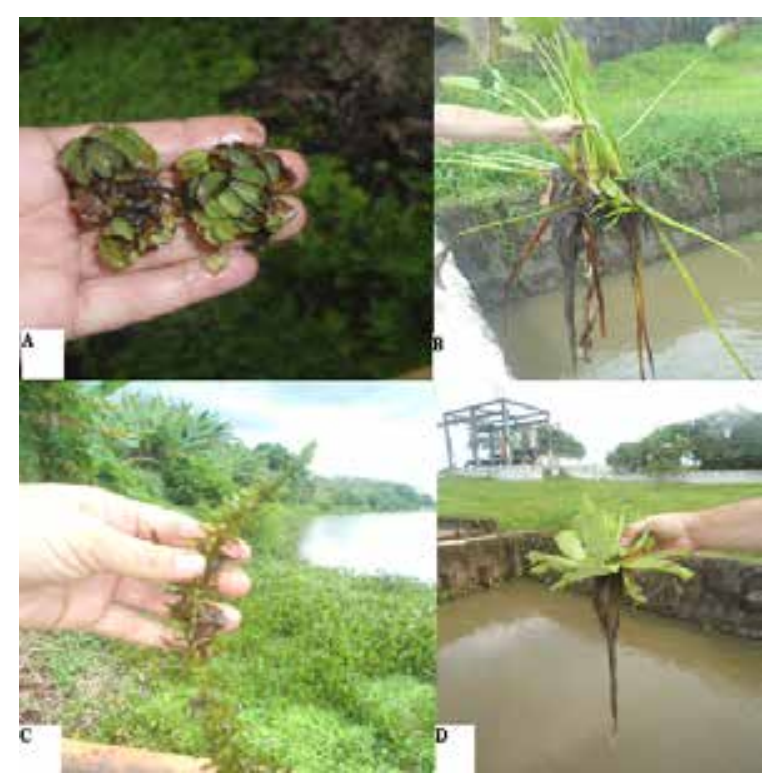

Figura 2: Espécies encontradas nos mananciais: (A) Salvinia auriculata, (B) Eichhornia crassipes, (C) Egeria densa, (D) Pistia stratiotes.

Dentre as espécies de plantas aquáticas identificadas, $S$. auriculata e $P$. stratioides apresentaram maior frequência de ocorrência nos mananciais estudados (60\%), seguidas por E. crassipes (40\%). Silva (2011) também observou essas espécies em maior frequência nos estudos realizados sobre a caracterização de macrófitas em reservatórios do estado de Pernambuco.

As correlações entre as variáveis físico-químicas analisadas e a presença ou ausência dos táxons de macrófitas são apresentadas na Tabela 2. 
Tabela 2: Correlação entre variáveis físico-químicas e a presença ou ausência dos táxons de macrófitas presentes nas unidades amostrais. Valores estatisticamente significativos em destaque.

\begin{tabular}{|c|c|c|c|}
\hline $\begin{array}{c}\text { Variáveis físico- } \\
\text { químicas }\end{array}$ & Salvinia auriculata & Pistia stratioides & Eichhornia crassipes \\
\hline Alcalinidade $\left(\mathrm{mgL}^{-1}\right)$ & $\mathrm{P}>0,05$ & $\mathrm{P}<0,05 ;$ rho $=0,45$ & $\mathrm{P}<0,05 ;$ rho $=0,45$ \\
\hline Cálcio $\left(\mathrm{mgL}^{-1}\right)$ & $\mathrm{P}<0,05 ;$ rho $=0,45$ & $\mathrm{P}<0,05 ;$ rho $=0,45$ & $\mathrm{P}<0,05 ;$ rho $=0,45$ \\
\hline Magnésio $\left(\mathrm{mgL}^{-1}\right)$ & $\mathrm{P}<0,001 ;$ rho $=0,77$ & $\mathrm{P}<0,05 ;$ rho $=0,45$ & $\mathrm{P}<0,05 ;$ rho $=0,45$ \\
\hline $\mathrm{pH}$ & $\mathrm{P}>0,05$ & $\mathrm{P}>0,05$ & $\mathrm{P}>0,05$ \\
\hline Condutividade $\left(\mu \mathrm{Scm}^{-1}\right)$ & $\mathrm{P}>0,05$ & $\mathrm{P}>0,05$ & $\mathrm{P}>0,05$ \\
\hline Turbidez $(\mathrm{NTU})$ & $\mathrm{P}<0,001 ;$ rho $=0,77$ & $\mathrm{P}<0,05 ;$ rho $=0,45$ & $\mathrm{P}<0,05 ;$ rho $=0,45$ \\
\hline Cor $(\mathrm{uH})$ & $\mathrm{P}<0,005 ;$ rho $=0,60$ & $\mathrm{P}>0,05$ & $\mathrm{P}>0,05$ \\
\hline Dureza $\left(\mathrm{mgL}^{-1}\right)$ & $\mathrm{P}<0,001 ;$ rho $=0,77$ & $\mathrm{P}<0,05 ;$ rho $=0,45$ & $\mathrm{P}<0,05 ;$ rho $=0,45$ \\
\hline Cloretos $\left(\mathrm{mgL}^{-1}\right)$ & $\mathrm{P}>0,05$ & $\mathrm{P}>0,05$ & $\mathrm{P}>0,05$ \\
\hline Sulfato $\left(\mathrm{mgL}^{-1}\right)$ & $\mathrm{P}<0,01 ;$ rho $=-0,54$ & $\mathrm{P}<0,001 ;$ rho $=-0,94$ & $\mathrm{P}<0,001 ;$ rho $=-0,94$ \\
\hline Amônia $\left(\mathrm{mgL}^{-1}\right)$ & $\mathrm{P}>0,05$ & $\mathrm{P}<0,005 ;$ rho $=-0,58$ & $\mathrm{P}<0,005 ;$ rho $=-0,58$ \\
\hline Nitrito $\left(\mathrm{mgL}^{-1}\right)$ & $\mathrm{P}<0,001 ;$ rho $=0,84$ & $\mathrm{P}<0,001 ;$ rho $=0,64$ & $\mathrm{P}<0,001 ;$ rho $=0,64$ \\
\hline Nitrato $\left(\mathrm{mgL}^{-1}\right)$ & $\mathrm{P}>0,05$ & $\mathrm{P}<0,005 ;$ rho $=-0,58$ & $\mathrm{P}<0,005 ;$ rho $=-0,58$ \\
\hline Fósforo $\left(\mathrm{mgL}^{-1}\right)$ & $\mathrm{P}<0,001 ;$ rho $=0,77$ & $\mathrm{P}<0,001 ;$ rho $=0,89$ & $\mathrm{P}<0,001 ;$ rho $=0,89$ \\
\hline
\end{tabular}

Observa-se correlação significativa entre $E$. crassipes e $P$. stratioides e as variáveis abióticas fósforo, amônia, nitrito, nitrato, sulfato, dureza, cálcio, magnésio, turbidez e alcalinidade. Presença de $S$. auriculata foi correlacionada significativamente com fósforo, nitrito, sulfato, dureza, cor, turbidez, cálcio, magnésio.

$\mathrm{Na}$ análise de nutrientes, o fósforo foi o parâmetro que apresentou maior correlação positiva para as três espécies de macrófitas: $P$. stratioides (rho $=$ 0,89 ), E. crassipes (rho $=0,89$ ) e S. auricualta (rho $=0,77$ ). Bornette e Puijalon (2011), em seu estudo de revisão, relatam uma relação positiva entre a ocorrência de espécies flutuantes e maiores concentrações de nitrogênio e fósforo.

Em relação às concentrações de amônia e nitrato, houve correlação negativa para a $P$. stratioides ( $r$ o $=-0,58)$ e $E$. crassipes ( $r$ o $=-0,58$ ), ou seja, a presença desses elementos inibe o crescimento dessas espécies, efeito também observado por Botino et al. (2014) em seus estudos.

Foi observada forte correlação negativa entre concentrações de sulfato e a presença de $P$. stratioides $($ rho $=-0,94)$ e E. crassipes ( $r h o=-0,94)$. De acordo com Bornette e Puijalon (2011), macrófitas de água doce podem apresentar baixa ou nenhuma tolerância à salinidade. Segundo Esteves (2011), o sulfato está entre os principais íons responsáveis pela salinidade da água, seu teor é fortemente influenciado pela formação geológica das bacias de drenagem e a proximidade com o mar, exercendo efeito negativo para o desenvolvimento dessas espécies.

S. auriculata é considerada uma espécie muito frequente em mananciais de água parada ou pouco movimentada, com grande potencial de cobertura superficial, bloqueando a passagem de luz solar e interferindo no metabolismo aquático. $P$. stratiotes é uma espécie que cobre totalmente o ambiente aquático, desenvolvendo-se rapidamente nos ambientes poluídos e provocando profundas alterações no ecossistema (ESTEVES, 2011).

A literatura sobre $E$. crassipes é extremamente extensa, dada a importância ecológica e sanitária assumida por essa espécie. Apresenta ampla distribuição em regiões tropicais e subtropicais. É nativa do Brasil, com distribuição extensiva no Norte e Nordeste, sendo particularmente abundante em Pernambuco (BOTINO et al., 2014). Esteves (2011) afirma que essa espécie é uma das 
macrófitas aquáticas mais comuns em ambientes eutrofizados e que, junto com Salvinia são as mais invasoras, tendo comprometido diversos usos da água em várias regiões quentes do mundo.

Com relação à influência das macrófitas sobre o tratamento de água, observou-se a obstrução da tubulação e comprometimento das bombas nas captações pela presença de Salvinia auriculata e Egeria densa, condição também reportada por Silva (2016) em seus estudos no reservatório de Guarapiranga, enquanto a S. auriculata foi encontrada em floculadores (Figura 3) e filtros (Figura 4) nas Estações de Tratamento de Água.

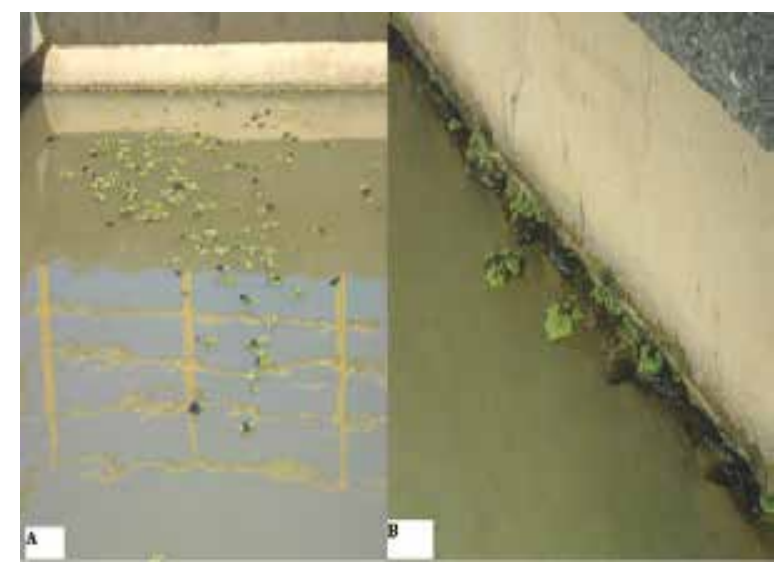

Figura 3: Presença de macrófitas na superfície (A) e paredes (B) dos floculadores em ETA.

Devido ao menor peso específico desses organismos, observou-se, durante a etapa de floculação, a flotação dos flocos contendo espécies de macrófitas (Figura 3a) e adesão desses organismos às paredes dos floculadores (Figura 3b). Com isso, as macrófitas passam por todas as etapas do tratamento e chegam aos filtros (Figura 4).

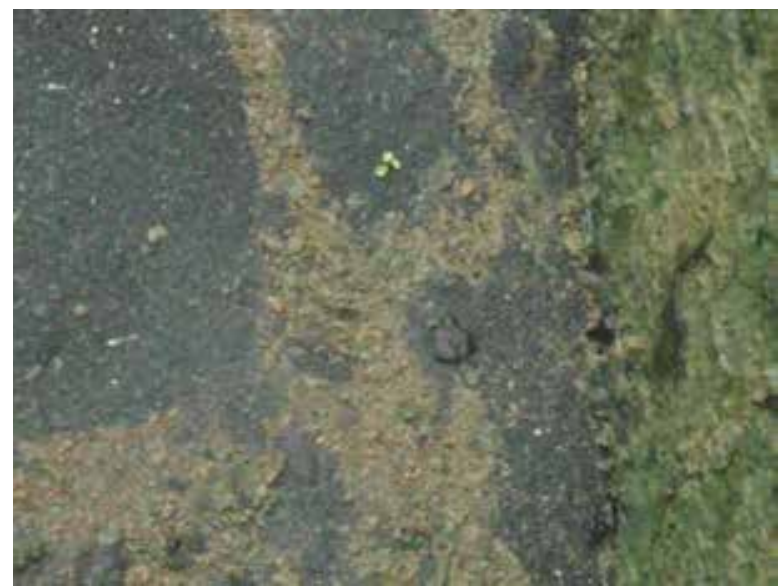

Figura 4: Vegetação na superfície do leito de areia e paredes do filtro.

A camada escura na superfície da areia (Figura 4) representa a vegetação morta que se deposita no fundo e é decomposta pelos organismos que vivem na superfície do meio filtrante, podendo causar obstrução do meio filtrante, redução da duração da carreira de filtração e consequente aumento do consumo de água para lavagem.

A partir de certo limite, o desenvolvimento da vegetação aquática pode ser considerado prejudicial aos usos múltiplos de ecossistemas aquáticos e, então, os métodos de controle ou manejo são necessários para minimizar o comprometimento do tratamento na ETA. As macrófitas aquáticas têm sido combatidas utilizando o controle mecânico, químico e biológico (PIETERSE e MURPHY,

Os métodos físicos ou mecânicos são os que apresentam a vantagem de não utilização de produtos ou organismos nocivos ou que persistem no ecossistema após sua introdução. No controle mecânico, as plantas precisam ser coletadas, transportadas e depositadas em local adequado, o que torna 
o processo oneroso e com eficácia de curto prazo, pois em pouco tempo os reservatórios são novamente colonizados. Frequentemente a equipe de manutenção da ETA realiza a raspagem do leito e limpeza das paredes dos filtros para remoção da vegetação. Esse procedimento possui maior eficiência em ambientes menores e mais rasos (THOMAZ, 2002). Outra alternativa adotada pela ETA foi a cobertura dos filtros com lonas (Figura 5), reduzindo a incidência de luz, evitando o efeito fotossintético para tentar controlar a proliferação da vegetação e algas. Do ponto de vista de manutenção do filtro, esta se apresentou como a meIhor opção, embora a redução da incidência de luz mesmo sendo eficiente fica inviabilizada em locais com elevada área superficial (DAMATO, 2016; NAKAMOTO, 2009).

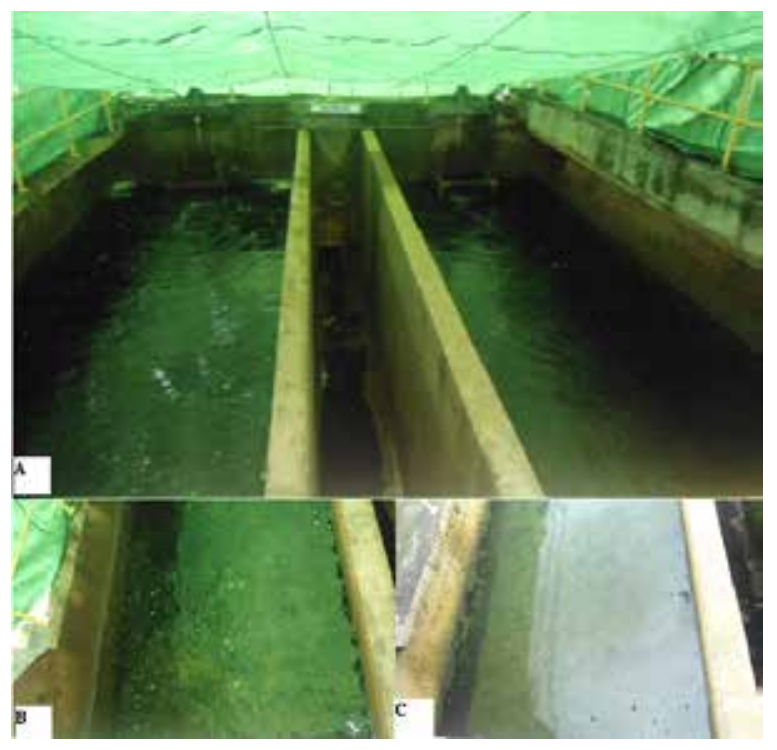

Figura 5: Filtro coberto com lona (A) e abaixo detalhe das paredes dos filtros cobertos (B) e sem cobertura (C).

Os métodos químicos são os menos utilizados, em face dos custos de aplicação e manejo desses produtos, segurança de uso e eficiência em longo prazo. O controle químico das macrófitas aquáticas pode ser feito com o uso de herbicidas. No Brasil, o fluridone é o único herbicida registrado para controle de macrófitas aquáticas do gênero Egeria (GARLICH et al., 2016).

O controle biológico tem aparecido como uma alternativa à aplicação de métodos químicos. Vários tipos de organismo, como fungos (BARRETO, 2000), insetos (HARLEY e FORNO, 1990) e peixes (PIETERSE e MURPHY, 1990), podem ser aplicados com esse intuito. Um dos problemas desse método é que, na maioria das vezes, utilizam-se espécies exóticas, que podem trazer severos prejuízos ambientais (THOMAZ, 2002).

A ETA avaliada no estudo tem recorrido frequentemente ao uso de métodos físicos e mecânicos para controle e manutenção de suas unidades

\section{CONCLUSÕES}

Dentre as espécies mais comuns encontradas nas captações que abastecem a região metropolitana do Recife, S. auriculata e P. stratioides ocorreram em maior frequência.

As concentrações de fósforo presentes nos mananciais foram correlacionadas positivamente (rho $=0,89)$ à presença das macrófitas encontradas (S. auriculata, E. crassipes e P. stratioides), sugerindo que o crescimento delas é influenciado pelo fósforo. Sendo assim, a remoção de fósforo em reservatórios a montante, restringindo o aporte de nutrientes e a influência antrópica, pode limitar as populações das macrófitas a jusante.

O processo de sedimentação observado na Barragem de Tapacurá, devido à disponibilidade de nutrientes, permite o avanço das plantas aquáticas, ocasionando a diminuição da velocidade da água, precipitação de partículas em suspensão e, consequentemente, reduz-se a vida útil do reservatório, podendo baixar sua capacidade de acúmulo de água.

A utilização de métodos mecânicos ou manuais tem sido a opção adotada pela unidade de tra- 
tamento para limitar a área de crescimento das plantas aquáticas e proteger as captações, além de ações frequentes de raspagem do leito de areia e limpeza das paredes das unidades para remoção da vegetação que chega à ETA.

O controle das plantas e a conservação da bacia a montante dos mananciais são decisivos para amenizar o processo de infestação das plantas observado nos reservatórios, que traz impactos diretos aos sistemas de tratamento.

\section{REFERÊNCIAS}

ALBERTONI, E.F.; PALMA-SILVA, C.; TRINDADE, C.R.T; FURLANETTO, L.M. (2014) Field evidence of the influence of aquatic macrophytes on water quality in a shallow eutrophic lake over a 13-year period. Acta Limnologica Brasiliensia, v. 26, n. 2, p. 176-185.

APHA.. Standard methods for examination of water and wastewater. 22nd ed., American Public Health Association, American Water Works Association and Water Environmental Federation, USA, 2012.

BARRETO, R. Biological control of neotropical aquatic weeds with fungi. Crop Protection, v. 19, n. 8, p. 697-703, 2000

BORNETTE, G.; PUIJALON, S. Response of aquatic plants to abiotic factors: a review. Aquatical Sciences, v. 73, p.1-14, 2011

BOTTINO, F;; CALIJURI, M.C.; MURPHY, K.J. Temporal and spatial variation of limnological variables and biomass of different macrophyte species in a Neotropical reservoir (São Paulo - Brazil). Acta Limnologica Brasiliensia, v.25, p. 387-397, 2014.

DAMATO, M. Microbiologia Aplicada a Operações e Processos de Engenharia Sanitária e Ambiental, 2008. Disponível em: http://www.brookepeig.com/downloads/Algas.pdf. Acesso em 04/04/2016.

DI BERNARDO, L. Algas e suas Influências na Qualidade das Águas e nas Tecnologias de Tratamento. Rio de Janeiro: ABES, 1995.

ESTEVES, F.A. Fundamentos de limnologia. 3.ed. Rio de Janeiro: Interciência - FINEP, 790 p., 2011.

GARLICH, N.; CRUZ, C.; SILVA, A.F.; CARRASCHI, S.P.; MALASPINA, I.C.; PITELLI, I.A.; BIANCO, S. Diquat associated with copper sources for algae control: Efficacy and ecotoxicology. Journal of Environmental Science and Health, v.51, n.4, p.17-29, 2016.
HARLEY, K. L. S.; FORNO, I. W. Biological control of aquatic weeds by means of arthropods. Aquatic weeds. The ecology and management of nuisance aquatic vegetation. New York: Oxford Science Publications. 177-185 p., 1990.

HOLMROOS, H.; HORPILLA, J.; NIEMISTÖ, J.; NURMINEM, L.; HIETANEN, S. Dynamics of dissolved nutrients among different macrophyte stands in a shallow lake. Limnology, v.16, p.31-39, 2015.

LIMA, L.L.F.; SILVA, S.S.L.; ZICKEL, C.S. Composição florística e chave de identificação das macrófitas aquáticas ocorrentes em reservatórios do estado de Pernambuco. Rodriguesia, v.62, n.4, p.1-7, 2011.

MEI, X.; ZHANG, X. Periphyton biomass response to phosphorus additions in an aquatic ecosystem dominated by submersed plants. Journal Environmental Protection, v.4, n.1, p. 83-90, 2013.

NAKAMOTO, N. Produza você mesmo uma água saborosa: sistema de purificação ecológica: revendo a tecnologia de produção da água potável. São Paulo. Editora Ferrari, 2009.

PIETERSE, A.H., MURPHY, K.J. Aquatic weeds. New York: Oxford Science Publications, 593 p., 1990.

RICHTER, C. A.; AZEVEDO NETO, J. M. Tratamento de Água: Tecnologia atualizada. 7 ed. São Paulo: Ed. Edgard Blücher Ltda, 332 p., 2007.

SILVA, S.S.L. Caracterização ecológica e estrutural de macrófitas em reservatórios no estado de Pernambuco. Tese (Doutorado em Biologia) - Universidade Federal Rural Pernambuco. 108 p., 2011.

SILVA, T.L.R. Análise da influência de variáveis meteorológicas e da hidrodinâmica sobre os padrões espaciais e temporais da qualidade da água e de florescimento de cianobactérias e macrófitas em um reservatório urbano. Tese (Doutorado em Ciências de Engenharia Ambiental) - Universidade de Engenharia de São Carlos. Universidade de São Paulo. 133 p., 2016.

THOMAZ, S.M. Fatores ecológicos associados à colonização e ao desenvolvimento de macrófitas aquáticas e desafios de Manejo. Planta Daninha. v..20, n. spe, p.21-33, 2002.

THOMAZ, S.M.; BINI, L.M. Ecologia e Manejo de Macrófitas Aquáticas. Editora da Universidade Estadual de Maringá. 341 p., 2003.

VON SPERLING, M. Introdução à Qualidade das Águas e ao Tratamento de Esgotos. Belo Horizonte. Departamento de Engenharia Sanitária e Ambiental, UFMG. 3a edição, v. 1. p. 452, 2005.

YADAV, A.K.; PATHAK, B.; FULEKAR, M.H. Rhizofiltration of Heavy Metals (Cadmium, Lead and Zinc) From Fly Ash Leachates Using Water Hyacinth (Eichhornia crassipes). International Journal of Environmental, v.4, n.1, p.179-196, 2015. 